\title{
An enormous pelvic tumor in a 46 -year-old woman with an elevated serum CA 125 level, what lies beneath it? Investigation of uterine tumors in postmenopausal women
}

\author{
Francesk Mulita, Elias Liolis, Dimitrios Kehagias, Levan Tchabashvili, Charalampos Kaplanis, \\ Fotios Iliopoulos, Ioannis Perdikaris, loannis Kehagias
}

Department of Surgery, General University Hospital of Patras, Rio, Greece

\begin{abstract}
Abdominal and pelvic pain with an associated pelvic mass is a very common emergency situation. There is always a management dilemma for most emergency physicians regarding these patients. A 46-year-old postmenopausal woman was admitted to our emergency department (ED) with complaints of massive abdominal distention. Abdominal and pelvis magnetic resonance imaging (MRI) was performed, which revealed a huge pelvic abdominal mass. All tumor markers were within normal limits. However, the ovarian cancer antigen (CA 125) level was elevated. As there was a strong suspicion of malignancy, the patient underwent total abdominal hysterectomy with bilateral salpingo-oophorectomy. Her final histopathology report was suggestive of uterine leiomyoma. Uterine leiomyomas are the most common benign uterine tumors in women. Surgical treatment is the gold standard, especially for older women with severe symptoms and no desire for future fertility. Although the combination of a pelvic tumor and a high-level of CA 125 arouses suspicion of gynecological malignancy, other benign conditions should always be considered in the differential diagnosis. There is limited evidence to support an association between elevated CA 125 levels and uterine fibroids so far. However, conditions such as the coexistence of adenomyosis and tumor size can affect the level of this marker in uterine fibroids.
\end{abstract}

Key words: menopause, uterine tumor, CA 125, leiomyoma.

\section{Introduction}

Abdominal and pelvic pain with an associated pelvic mass is a very common emergency situation. There is always a management dilemma for most emergency physicians regarding these patients. Physicians encounter difficulties in differentiating a potential surgical problem such as torsion of an enlarged ovary and a non-surgical pathology such as uterine leiomyoma in a physical examination. Different types of imaging examinations, e.g. ultrasonography (US), computed tomography (CT), and/or magnetic resonance imaging (MRI), are the gold standard imaging tools used to differentiate pelvic masses in women presenting with abdominal and pelvic pain to the emergency department (ED) [1].

\section{Case report}

A 46-year-old postmenopausal woman was admitted to our ED with complaints of massive abdominal distention which started gradually 30 days ago. She also complained of difficulty in breathing and ambulation. There were no other gastrointestinal, gynecologi- cal, or urinary symptoms. The woman had a significant past medical history of hypertension. Her family history is negative for ovarian and breast cancer in first-degree relatives. On physical examination, the patient's abdomen was distended. Blood analysis revealed hemoglobin of $13.9 \mathrm{~g} / \mathrm{dL}, 8280$ leukocytes, and 226000 platelets. All tumor markers were within normal limits. However, the ovarian cancer antigen (CA 125) level was $260.9 \mathrm{U} / \mathrm{mL}$ (normal range: $0-35 \mathrm{U} / \mathrm{mL}$ ). Abdominal and pelvis MRI was performed, which revealed a huge pelvic-abdominal mass measuring approximately $22 \times 15 \times 12 \mathrm{~cm}$ (Fig. $1 \mathrm{~A}, \mathrm{~B}, \mathrm{C}$ ). As there was a strong suspicion of malignancy, the patient underwent total abdominal hysterectomy with bilateral salpingo-oophorectomy. The enormous lobulated tumor weighing $6.7 \mathrm{~kg}$ had a thick wall with solid and cystic areas (Fig. 1 D). Her final histopathology report was suggestive of uterine leiomyoma. The patient had no intra-operative or postoperative complications. On day 5 after the surgery, the patient was discharged home. Three months following the surgery, the CA 125 levels had regressed to the normal range. The patient has since been symptom-free, and the CA 125 levels have also remained normal. 

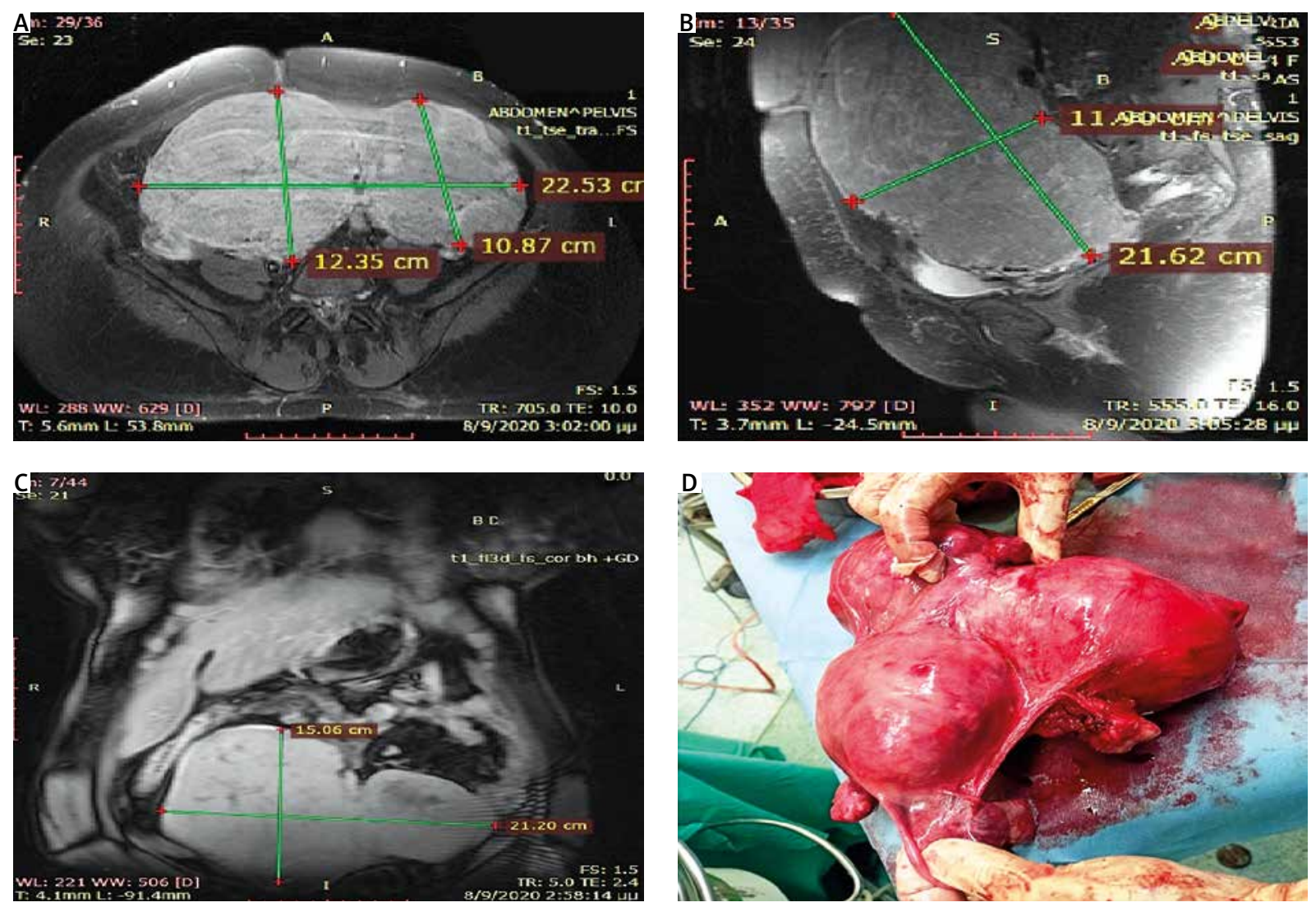

Fig. 1. A, B, C) magnetic resonance imaging images show 2 large subserosal fibroids arising from the anterior uterus, D) complete resection of the mass that weighed $6.7 \mathrm{~kg}$ and measured approximately $22 \times 15 \times 12 \mathrm{~cm}$

\section{Discussion}

Menopause is defined as the absence of menstrual cycles for at least twelve consecutive months, indicating the end of the reproductive age for women as they are not able to get pregnant naturally $[2,3]$ Menopause is a natural biological event, but the mean age can differ between women and it can be affected by several parameters, e.g. nationality, diet, other diseases. Menopause is also associated with hormonal changes. More specifically, the levels of estrogen, progesterone, follicle-stimulating hormone (FSH), and other hormones are affected. Early menopausal transition causes fluctuations in estrogen but eventually its production declines completely (last post-menopause stage). The same pattern is observed for FSH and progesterone [2]. These hormonal changes are associated with various symptoms such as hot flushes, night sweats, etc. [4]. Studies also have demonstrated a potential role of menopause onset with hormone-related cancers such as ovarian, endometrial, and breast cancer as late menopause means higher hormone levels for women and longer exposure to estrogens. According to one hypothesis, estrogens play a mitogenic role but mitotic activity increases the probability of DNA damage resulting in a mutation that can lead to the transformation of endometrial cells and tumor formation [2, 3, 5]. Therefore, a uterine tumor is a phenomenon usually observed in postmenopausal women.

Abnormal uterine bleeding is the first sign that leads postmenopausal women to seek gynecological advice. Bleeding may be due to a tumor, but many other diseases also can cause it $[6,7]$. Therefore, it is very important to proceed with the right diagnosis. The first step is the physical examination that involves a pelvic examination to check for lumps that should be further checked. After that, the first tool that can be used is the transvaginal ultrasound (TVUS), an imaging technique in which the doctor inserts an ultrasound probe into the vaginal canal to get images and to examine the organs of the female reproductive tract [6]. It has been observed that TVUS has $97 \%$ sensitivity and $74 \%$ specificity for the detection of endometrial abnormalities [8]. If any abnormality is detected, the next step is to proceed with biopsy. During this procedure, a sample of cells is removed from the inside lining of the uterus and it is examined using a microscope. If the biopsy shows endometrial cancer, the appropriate treatment should be given. However, if the biopsy is normal or non-diagnostic, other techniques can be used to investigate the cause of bleeding. More specifically, color Doppler can be used to differentiate between benign and malignant pathologies, and saline contrast hysterosonography is 
a technique used to evaluate the uterine cavity of postmenopausal women $[6,8]$. Finally, hysteroscopy with biopsy is used when the biopsy results are unclear or enough tissue cannot be removed. During this procedure, the doctor examines the inside of the cervix and uterus using a hysteroscope that resembles a small microscope [9]. It is also considered a safe technique and it has $100 \%$ sensitivity and $97 \%$ specificity for the detection of endometrial cancer [8].

As the most common symptom of a uterine tumor is abnormal uterine bleeding, it is very important to differentiate endometrial cancer at early stages from endometrial hyperplasia and endometrial polyps and also to differentiate advanced stages of cancer from uterine sarcoma or uterine lymphoma [10, 11]. Endometrial hyperplasia is an overgrowth of the endometrium which usually causes heavy or abnormal bleeding. Though it can increase the risk of endometrial cancer, it is very important to diagnose it accurately in order to proceed with the appropriate therapy. Therefore, an endometrial biopsy is the best tool to differentiate hyperplasia from cancer [12]. Endometrial polyps are localized hyperplastic overgrowths that occur in the endometrium, and their prevalence is higher among women with increasing age [13]. Most polyps are benign, in contrast with cancer tumors. Transvaginal US is usually the first tool for diagnosis, but it seems that color Doppler is more effective in distinguishing benign and cancerous tumors. However, the gold standard is hysteroscopy with guided biopsy [8]. Another disease that should be differentiated is uterine sarcomas, rare gynecologic tumors which are usually diagnosed in postmenopausal women between 50 and 70 years old. However, their diagnosis is extremely challenging. Imaging tools are not very helpful to distinguish sarcoma from endometrial hyperplasia or carcinoma, but it seems that endometrial biopsy is the best choice [14]. Finally, uterine lymphomas usually cause uterine bleeding and abdominal pain, but it is very important to distinguish them from other diseases such as endometrial cancer as they require different treatment options. Uterine lymphomas are rare malignancies (usually non-Hodgkin) of the female reproductive system and they are usually diagnosed using laparoscopy and biopsy [15].

Uterine leiomyomas (also known as fibroids) are the most common uterine tumors in women. Although the exact pathophysiology behind the development of these benign tumors remains unclear, they commonly result in severe symptoms such as abnormal bleeding, pelvic pain, pelvic masses, infertility, anemia, bulk symptoms and obstetric complications [16, 17].

Treatment options mainly involve surgical interventions, but the choice of treatment is individualized based on patient's age, size and location of the lesions, severity of the symptoms, and desire for future fertility. Hysterectomy is the gold standard of leiomyoma treatment, especially for women who do not wish to con- ceive and/or women aged above $40-50$ years, as it is immediately curative of the symptoms and can prevent their recurrence [18]. Our patient underwent surgery, as she was symptomatic and had completed having children. In addition, malignancy could not be excluded.

Cancer antigen is mostly used as a relatively specific marker for ovarian cancer. According to the literature, the combination of a pelvic tumor and a high level of CA 125 arouses suspicion of a gynecological malignancy, but other benign conditions such as menstruation, endometriosis, and pelvic inflammatory diseases should always be considered in the differential diagnosis, particularly in pre-menopausal women [19-21]. Although there is limited evidence to support an association between elevated CA 125 levels and uterine fibroids, abnormally high levels have been reported in a small number of patients. Furthermore, coexistence of adenomyosis and tumor size can affect the level of this marker in uterine fibroids. A large leiomyoma may be responsible for CA 125 elevations through an indirect mechanism such as peritoneal irritation [22].

\section{Conclusions}

Uterine leiomyomas are the most common benign uterine tumors in women. Surgical treatment is the gold standard, especially for older women with severe symptoms and no desire for future fertility. Although the combination of a pelvic tumor and a high level of CA 125 arouses suspicion of a gynecological malignancy, other benign conditions should always be considered in the differential diagnosis. There is limited evidence to support an association between elevated CA 125 levels and uterine fibroids so far. However, conditions such as coexistence of adenomyosis and tumor size can affect the level of this marker in uterine fibroids.

\section{Acknowledgement}

Informed consent was obtained from the patient for the publication of this article.

\section{Disclosure}

The authors report no conflict of interest.

\section{References}

1. Imai A, Ichigo S, Takagi H, et al. Pelvic tumors with normal-appearing shapes of ovaries and uterus presenting as an emergency (review). Oncol Lett 2012; 4: 10-14

2. Dunneram Y, Greenwood DC, Cade JE. Diet, menopause and the risk of ovarian, endometrial and breast cancer. Proc Nutr Soc 2019; 78: 438-448.

3. Wu Y, Sun W, Liu H, Zhang D. Age at menopause and risk of developing endometrial cancer: a meta-analysis. Biomed Res Int 2019; 2019: 8584130 . 
4. Kotsopoulos J. Menopausal hormones: definitive evidence for breast cancer. Lancet 2019; 394: 1116-1118.

5. Rodriguez AC, Blanchard Z, Maurer KA, Gertz J. Estrogen signaling in endometrial cancer: a key oncogenic pathway with several open questions. Horm Cancer 2019; 10: 51-63.

6. Amant F, Moerman P, Neven P, Timmerman D, van Limbergen E, Vergote I. endometrial cancer. Lancet 2005; 366: 491-505.

7. Mulita F, Iliopoulos F, Kehagias I. A rare case of gastric-type mucinous endocervical adenocarcinoma in a 59-year-old woman. Prz Menopauz 2020; 19: 147-150.

8. Otify M, Fuller J, Ross J, Shaikh H, Johns J. Endometrial pathology in the postmenopausal woman - an evidence based approach to management. Obst Gynaecol 2015; 17: 29-38.

9. Garuti G, Angioni S, Mereu L. et al. Hysteroscopic view with targeted biopsy in the assessment of endometrial carcinoma. What is the rate of underestimatated diagnosis? The results of a multicenter Italian trial. Gynecol Surg 2020; 17: 10.

10. Hippisley-Cox J, Coupland C. Identifying women with suspected ovarian cancer in primary care: derivation and validation of algorithm. BMJ 2011; 344: d8009.

11. Mulita F, Iliopoulos F, Plachouri KM, et al. Uterine leiomyoblastoma. BMJ Case Rep 2021; 14: e241533.

12. Horn LC, Meinel A, Handzel R, Einenkel J. Histopathology of endometrial hyperplasia and endometrial carcinoma: an update. Ann Diagn Pathol 2007; 11: 297-311.

13. Nijkang NP, Anderson L, Markham R, Manconi F. Endometrial polyps: pathogenesis, sequelae and treatment. SAGE Open Med 2019; 7 : 2050312119848247.

14. Bužinskienė $D$, Mikènas S, Drąsutienė G, Mongirdas M. Uterine sarcoma: a clinical case and a literature review. Acta Med Litu 2018; 25: 206-218.

15. Samama M, van Poelgeest $M$. Primary malignant lymphoma of the uterus: a case report and review of the literature. Case Rep Oncol 2011; 4: $560-563$.

16. Donnez J, Dolmans MM. Uterine fibroid management: from the present to the future. Hum Reprod Update 2016; 22: 665-686.

17. Sabry M, Al-Hendy A. Medical treatment of uterine leiomyoma. Reprod Sci 2012; 19: 339-353.

18. Mas A, Tarazona M, Dasí Carrasco J, et al. Updated approaches for management of uterine fibroids. Int J Womens Health 2017; 9: 607-617.

19. Asher V, Hammond R, Duncan TJ. Pelvic mass associated with raised CA 125 for benign condition: a case report. World J Surg Oncol 2010; 8: 28.

20. Mulita F, Tavlas P, Maroulis I. A giant ovarian mass in a 68 -year-old female with persistent abdominal pain and elevated serum CA-125 level. Prz Menopauz 2020; 19: 108-110.

21. Mulita F, Oikonomou N, Tchabashvili L, et al. A giant ovarian mucinous tumor in a 58-year-old postmenopausal patient with persistent abdominal pain and high serum levels of CA 19-9. Pan Afr Med J 2020; 37: 76.

22. Babacan A, Kizilaslan C, Gun I, et al. CA 125 and other tumor markers in uterine leiomyomas and their association with lesion characteristics. Int J Clin Exp Med 2014; 7: 1078-1083. 